\title{
Rehabilitation ist keine Light-Pflege und auch kein Hotel-Angebot
}

\author{
Willy Oggier \\ Dr. oec. HSG, Gesundheitsökonomische Beratungen AG und Präsident SW!SS REHA*, Küsnacht
}

SW!SS REHA ist ein Verband von Rehabilitationskliniken in der Schweiz, der für seine Mitglieder verbindliche Qualitätskriterien definiert hat. Verschiedene Kantone stützen ihre Rehabilitationsplanungskriterien auf diese Vorgaben ab.
In jüngerer Zeit mehren sich Rundumschläge zur Rehabilitation. So bezeichnete etwa die Direktorin der Zürcher Pflegezentren diese als "teure» Übergangslösung für Hochbetagte (hotel revue, 18. Dezember 2015). Eine solche Sichtweise darf nicht unwidersprochen bleiben. Denn sie verkennt, dass die heutige Situation der Trennung von Rehabilitationsleistungen von Kur- und/oder Akut- und Übergangspflege-Leistungen vom Gesetzgeber gewollt war. Jede Leistung hat ihre unterschiedliche Berechtigung und daher ist es auch folgerichtig, dass entsprechend unterschiedliche Finanzierungsregeln und insbesondere Kostenübernahmen durch die soziale Krankenversicherung erfolgen.

Seit Jahren existierten von SW!SS REHA Kriterien für die stationäre Rehabilitation, welche laufend ergänzt und weiterentwickelt wurden. Neben allgemeinen Rehabilitationskriterien, die alle Kliniken erfüllen müssen, wurden detailliertere Anforderungen für die geriatrische, die internistische und onkologische, die kardiovaskuläre, die muskuloskelettale, die neurologische, die pädiatrische, die paraplegiologische, die psychosomatische und die pulmonale Rehabilitation entwickelt. Rund 50 Prozent der Rehabilitationskliniken der Schweiz erfüllen die SW!SS REHA-Kriterien und werden durch die entsprechenden Assessoren auch periodisch reevaluiert.

Nachdem die Bedeutung der ambulanten und teilstationären Rehabilitation in den letzten Jahren an Bedeutung gewonnen hat, wurden entsprechende Kriterien auch für diese Rehabilitationsbereiche definiert. Sie sollen die Basis dafür bilden, die Spreu vom Weizen zu trennen und damit Pseudo-Anbieter transparent offenzulegen.

Die entwickelten SW!SS REHA-Kriterien sind auf der Website www.swissreha.com einsehbar. Erste Erfolge der langjährigen Qualitäts-Arbeiten von SW!SS REHA werden insbesondere in der Deutsch- und Südschweiz sichtbar. So hat etwa der Kanton Tessin für die Vergabe von Rehabilitations-Leistungsaufträgen die Auflage gemacht, dass die entsprechenden Kliniken die SW!SS REHA-Kriterien erfüllen müssen. Diese Kriterien können nur erfüllt werden, wenn auch das Assessment

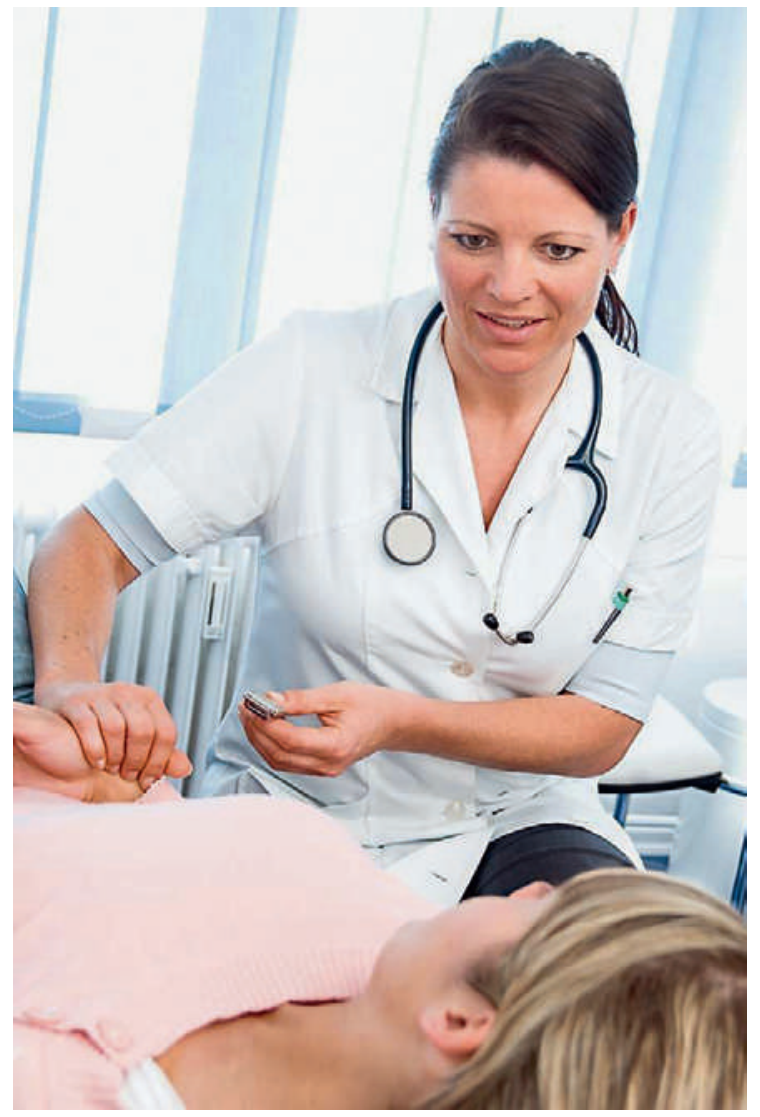

Eine Rehabilitation beinhaltet auch ärztliche Leistungen, die Übergangspflege beispielsweise in der Regel nicht.

durch die SW!SS REHA erfolgreich durchgeführt worden ist. Daher erfolgte beispielsweise die Gewährung des Leistungsauftrags für psychosomatische Rehabilitation nicht an eine psychiatrische, sondern an eine entsprechende Rehabilitationsklinik.

Auch weitere Gemeinwesen beginnen, die Qualitätskriterien für ihre Arbeit zu nutzen. So hat etwa die grösste Schweizer Stadt, Zürich, im Rahmen ihrer Ausschreibung von Rehabilitationsleistungen für ihr eigenes Gesundheits-Netzwerk ausschliesslich SW!SS REHA-Mitglieder zur Bewerbung zugelassen. Pflegeoder Physiozentren werden diesem Anspruch nicht gerecht, auch wenn sie vermeintlich billigere Tarife anbieten. Denn wie formulierte die Initiative der Baden- 


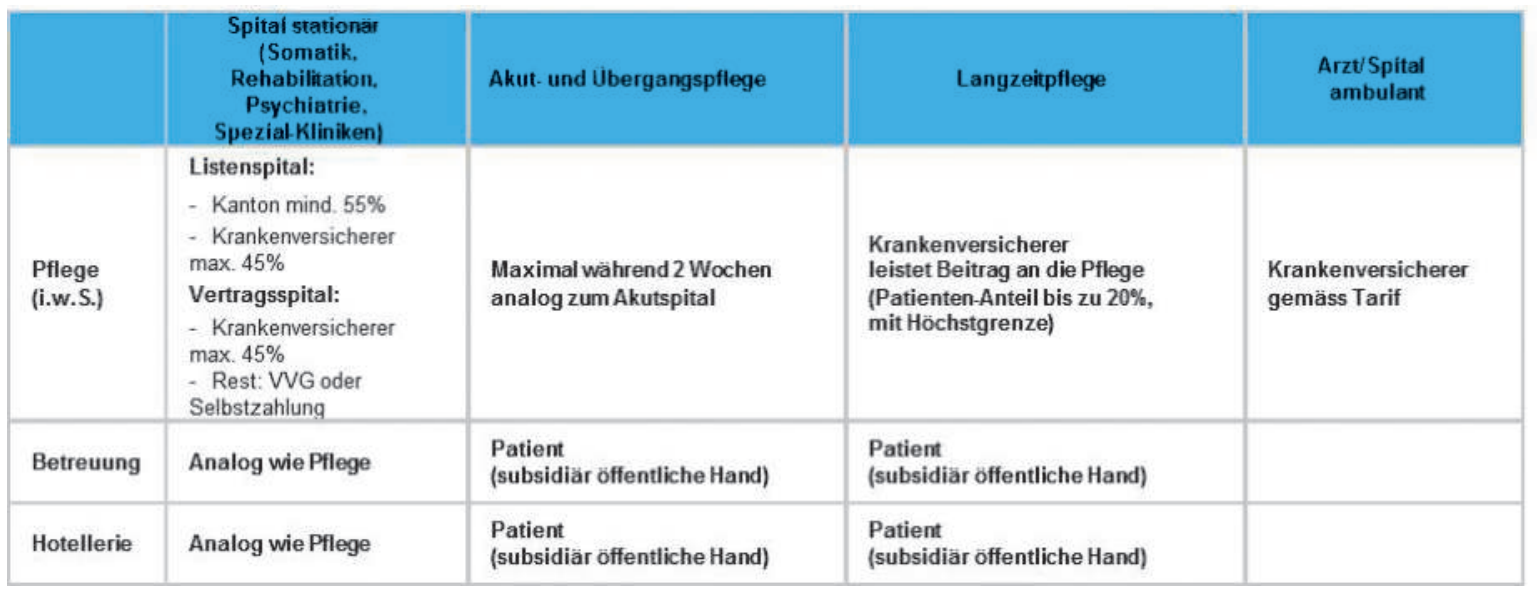

Tabelle 1: Finanzierungsregeln nach Krankenversicherungsgesetz (KVG).

Württembergischen Krankenhausgesellschaft doch unlängst so schön: «Umsonst ist keine Reha!»

\section{Abgrenzung: Rehabilitation - Übergangs- pflege - Kur - ambulante Therapie ${ }^{1}$}

Leistungen der Akut- und Übergangspflege, die sich im Anschluss an einen Spitalaufenthalt als notwendig erweisen und die im Spital ärztlich angeordnet werden, werden von der obligatorischen Krankenversicherung und vom Wohnkanton des Versicherten während längstens zwei Wochen nach den Regeln der Spitalfinanzierung vergütet. Dabei beziehen sich diese Regeln ausschliesslich auf die pflegerischen Leistungen, nicht jedoch auf die Kosten für Betreuung und Hotellerie. Diese Kosten sind vom Patienten als Selbstzahler oder durch eine allfällig vorhandene Zusatzversicherung zu bezahlen.

Übergangspflege findet dann statt, wenn

- die medizinisch oder psychiatrisch pflegerische Situation der Patienten stabil ist;

- ein Austritt aus dem Akutspital stattfinden soll;

- weiterhin mobilisierende und somatische oder psychiatrische Pflege notwendig ist, die ein Spitalarzt verordnet, und diese im Vordergrund steht;

- kein Langzeitpflegeaufenthalt absehbar ist;

- keine Indikation für eine Rehabilitation vorliegt;

- die Übergangspflege zeitlich begrenzt ist;

- der Wille des Patienten zur Übergangspflege und zur Wiedererlangung der Autonomie und Selbstversorgung vorliegt.

Kapitels basieren auf SW!SS REHA (Hrsg.)

Qualitäts- und Leistungskriterien für die ambulante und

teilstationäre

Rehabilitation, Stand vom 21. Oktober 2015.

Übergangspflege verfügt in der Regel über keine ärztliche Leistung. Es besteht in der Regel auch keine Therapie-Programm-Akkreditierung als Grundlage. Bzgl. der Finanzierungsströme nach KVG kann die Situation der Akut- und Übergangspflege wie in Tabelle 1 dargestellt werden.

Bei Kuren stehen in der Regel die Vorsorge bzw. Stärkung einer (geschwächten) Gesundheit oder die Unterstützung der Genesung bei Krankheiten und Leiden verschiedener Art durch die Anwendung ortspezifischer Heilmittel (z.B. Quellen, Peloide, Klima, Meerwasser) im Rahmen des Aufenthalts in einem Kurort oder Heilbad im Vordergrund. Gemäss KVG wird für Kurverfahren ein Beitrag von 10 Franken pro Tag (pauschal) für maximal 21 Tage pro Kalenderjahr entrichtet, zuzügl. Kosten für Arzt, Medikamente und Physiotherapien

Ambulante Therapieangebote (monodisziplinäre Einzelpraxen oder auch "Therapie-Zentren») unterscheiden sich von ambulanten Rehabilitationszentren insbesondere dadurch, dass sie keine interdisziplinär koordinierten Dienste anbieten bzw. nicht unter ärztlicher Leitung stehen.

\section{Abgrenzung: stationäre - teilstationäre - ambulante Rehabilitation}

Es ist grundsätzlich zwischen stationärer, teilstationärer und ambulanter Rehabilitation im Sinne eines abgestuften Leistungskonzeptes zu unterscheiden. Es bestehen Gemeinsamkeiten dieser drei Stufen hinsichtlich des Verständnisses von Rehabilitation und insbesondere des interdisziplinären Ansatzes sowie des Denkens in den Kategorien der International Classification of Functioning (ICF).

\section{Ambulante interdisziplinäre Rehabilitation}

Die ambulante Rehabilitation grenzt sich von einer monodisziplinären und oftmals eindimensional ausgerichteten Behandlungsmodalität wie beispielsweise 
einer ambulanten Physio- oder Ergotherapie respektive Logopädie ab. Die ambulante interdisziplinäre Rehabilitation vereint rehabilitationsmedizinisch relevante Berufsgruppen wie Physiotherapie, Ergotherapie, Logopädie, Neuropsychologie, Sozialdienst und ärztliche Leistungen unter einem Dach. Es werden in regelmässigen Teamsitzungen gemeinsame Ziele festgelegt. Der Verlauf wird dokumentiert und von einem Rehabilitationsarzt koordiniert. Es finden mehrere Therapieeinheiten pro Tag unter dem gleichen Dach und unter koordinierter, strukturierter Organisation statt. Zudem sind die Funktionseinschränkungen der betroffenen Personen so ausgeprägt, dass ein monodisziplinärer Ansatz mit zwei bis drei Behandlungsstunden pro Woche nicht ausreichend ist.

Die ambulante Rehabilitation ist vielfältiger als die stationäre oder teilstationäre Rehabilitation und kennt auch Spezialgebiete wie die Rehabilitation von Menbehinderungen. Hingegen ist der Schweregrad von Krankheiten und der Grad der Funktionseinschränkungen der Patienten in der ambulanten Rehabilitation - und somit die Behandlungsintensität - geringer als in der stationären oder teilstationären Rehabilitation.

\section{Teilstationäre Rehabilitation}

Die teilstationäre Rehabilitation grenzt sich ebenso wie die ambulante Rehabilitation von einer monodisziplinären, eher eindimensional ausgerichteten Behandlungsmodalität ab. Im Gegensatz zur ambulanten Rehabilitation ist die teilstationäre Rehabilitation stärker an die stationären Behandlungskonzepte angelehnt und weist in der Regel höhere Behandlungsintensitäten pro Woche auf. Dennoch ist der Schweregrad von Krankheiten und der Grad der Funktionseinschränkungen bei einer Gesamtbetrachtung des einzelnen Patienten in der teilstationären Rehabilitation geringer als in der stationären Rehabilitation.

Die wichtigen Definitionen wie der Rehabilitationsbedarf, die Rehabilitationsfähigkeit, das Rehabilitationspotential, die Rehabilitationsprognose sowie die generellen Ziele der Rehabilitation gelten gleichermassen für die ambulante und teilstationäre Rehabilitation.

Die ambulante und teilstationäre Rehabilitation können grundsätzlich in folgenden Situationen in Betracht kommen:

Dr. oec. HSG Willy Oggie

Weinhaldenstrasse 22

CH-8700 Küsnacht

gesundheitsoekonom.

willyoggier[at]bluewin.ch

- Bei fehlender Spitalbedürftigkeit anstelle einer stationären Rehabilitationsmassnahme als eigenständiges interdisziplinäres Konzept bei Patienten, bei schen mit Seheinschränkungen oder Hör- oder Sprach-

denen die Schwere von Krankheiten bzw. der Grad an Funktionseinschränkungen weniger stark ausgeprägt ist;

- zur Verkürzung einer stationären Rehabilitationsmassnahme dank ambulanter Fortsetzung eines stationär begonnenen Rehabilitationsprogramms;

- im Anschluss an eine stationäre Rehabilitationsmassnahme.

Für den Entscheid zwischen einer stationären, teilstationären oder ambulanten Rehabilitation ist die Spitalbedürftigkeit (Schweregrad der Krankheiten und Ausmass der Funktionseinschränkung, Therapieintensität, Unselbständigkeit, Pflege- und Betreuungsbedarf, sozialmedizinischer Kontext) ein wichtiges Kriterium. An dieser Schnittstelle kommt dem Rehabilitationsmediziner eine bedeutende Funktion zu.

\section{Fazit}

Es ist offensichtlich, dass Pflegezentren oder ähnliche Anbieter wie beispielsweise die in der Westschweiz stark verbreiteten Centres de thérapie et de réadaptation (CTR) die Anforderungen an eine wirksame, zweckmässige und wirtschaftliche Rehabilitation, wie sie das KVG fordert, weitgehend nicht erfüllen können. Dies gilt gerade auch für ältere, an Mehrfacherkrankungen leidenden Menschen. In verschiedenen Rehabilitations-Disziplinen ist das Durchschnittsalter der Patienten in vielen Kliniken über 70 Jahre. Verschiedene unter ihnen verfügen über Beatmungsplätze. Dies macht deutlich, dass die Rehabilitationskliniken sehr wohl auf komplexe Situationen vorbereitet sind. Dass Pflegezentren und die Hotellerie-Branche vermehrt in die Rehabilitation vordringen wollen, dürfte eher andere Gründe haben. Mit der vorgesehenen neuen leistungsorientierten Tarifstruktur nach KVG dürften Rehabilitationskliniken bei Patienten mit höherem Schweregrad künftig Aussicht auf eine höhere Abgeltung haben. Durch eine Um-Definition der Leistungsaufträge über Begriffs-Verwirrungen scheint man sich bei den Pflegezentren einen erweiterten $\mathrm{Zu}$ gang zu den Finanzierungsquellen der sozialen Krankenversicherung verschaffen zu wollen. Dies ist aber nicht im Sinn des Gesetzgebers. Denn die Mengenausweitung durch Pseudo-Anbieter wäre damit vorprogrammiert.
Bildnachweis

(c) MEV Verlag GmbH, Germany 